\title{
Super Resolution - An image Enhancement and Upscaling Technique for Satellite Imagery
}

\author{
Durai Pandurangan, Ramakrishna MM, Gopala Krishna R, Alemayehu Kebede
}

\begin{abstract}
Satellite imagery is the phenomenon of extracting information from the images takes by satellite which is owned by the government or business throughout the world. In other words, we can say satellite imagery is the spaceborne photography which zooms enough to take photos of the surface of the earth and other planets of the solar system. Most of the time images captured are not in sync which makes it really complex to study. Nowadays satellites are used by the government for patrolling border security and terrorist activities. To clarify such extra zoomed image for human visibility 'super-resolution"' was introduced. Super-resolution is an image processing based resolution enhancement technique which improves the details available in an image. An image with power order of resolution is taken an upscaled to its higher-order using multi-surface fitting and image pixel transformations. Our proposed paper is to process the image in the proper order to enhance the image quality so that we can increase the zooming capability of satellite imagery.
\end{abstract}

Index Terms: Satellite Imagery, Multisurface Fitting,

\section{INTRODUCTION}

Obtaining an image of higher-order resolution is the requirement of time. Almost in all image related domains like medical imaging, satellite imaging, defence sector, researches, remote sensing and space science the requirement of higher-order resolution is critical. The quality of image degrades due to several artefacts blurring, hazing image noise, aliasing etc. these artefacts are possible to be avoided till the maximum extents by using digital filter and transformation techniques of the pixels.

If we want to increase the quality of the image by using batter lenses and camera mechanism then its costs in millions. Even assembling and installing that camera is highly complex which needs an expert supervisor and the biggest space. In space aviation, this biggest size is another problem. In another domain like medical imaging, defence the cost is the parameter. After several types of research, it was considered that it is best to have digital filtration and transformation techniques to enable a good camera into a

great with image resolution enhancement capabilities. The super solution enables the image enhancement feature to the cameras without physically increasing its size or

Revised Manuscript Received on December 30, 2019.

Mr. Durai Pandurangan, Lecturer in Department of Electrical and Computer Engineering, Wachemo University (WCU), Hossana, Ethiopia.

Mr.Ramakrishna MM, Pursuing PhD at Dr.M.G.R Educational and Research Institute in the field of Sensor.

Mr. Gopala Krishna R, Lecturer in Department of Electrical and Computer Engineering, Wachemo University (WCU), Hossana, Ethiopia.

Mr. Alemayehu Kebede, Lecturer in Department of Electrical and computer Engineering,Wachemo University (WCU), Hossana, Ethiopia. Super-Resolution (SR)

weight. In the economical perspective also it's way cheaper. Super Resolution and Blur Deconvolution (BD) are two great techniques of image upscaling but there is a big difference between them. Blur Deconvolution (BD) concentrates towards deblurring and noise separation but super-resolution also enhances the antialiasing capabilities of the image.
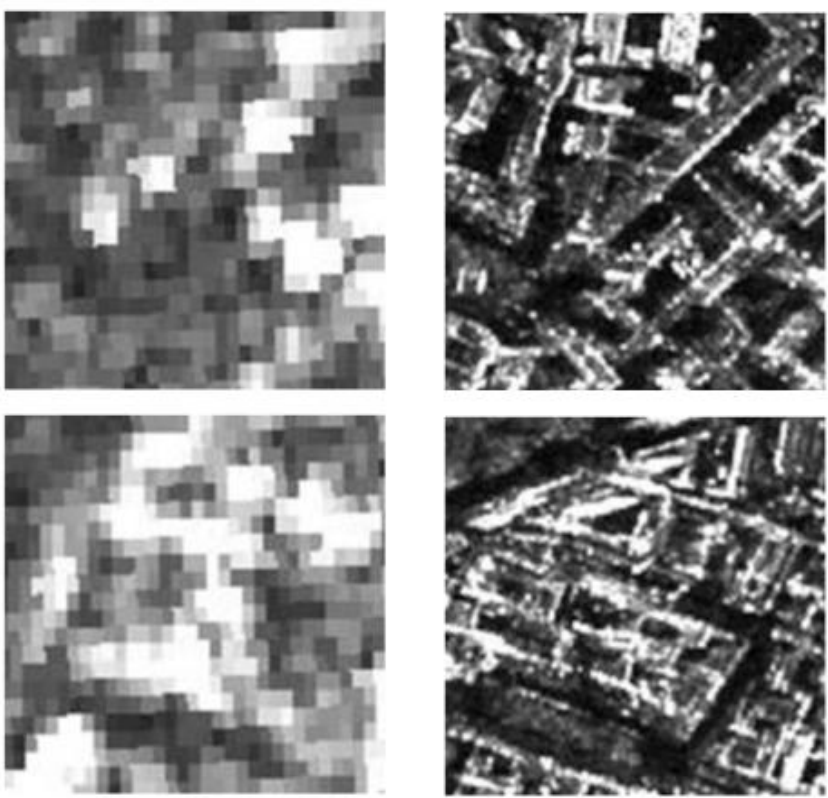

Fig. 1.1 - Super Resolution with Multiple Enhancements on base image

The image provided in the Blur Deconvolution process remains almost same size during input and output processes but in Super-Resolution output images are always bigger compared to the input image. The quality enhancement and pixel transformation always increase the size of the image in Super-Resolution. The overall Blur is mitigated much extensively in Super-Resolution compared to Blur Deconvolution (BD).

\section{LITERATURE SURVEY}

[1]. Sina Farsiu, Michael Elad and Peyman Milarfar explained in their paper 'A practical approach to Super Resolution' that Super Resolution can be used for several applications but their paper explains about the issues which come in Super-Resolution. The major two issues are Reconstruction accuracy and computation efficiency which means if we apply Super Resolution concept then lower pixel or low resolution (LR) pixel are boosted up for High Resolution (HR) pixels.

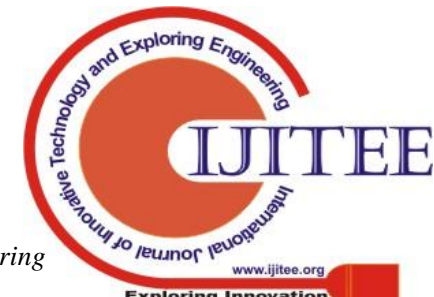




\section{Super Resolution - An image Enhancement and Upscaling Technique for Satellite Imagery}

During the reconstruction, the rearrangement of the pixels should be very accurate. The errors generated by types of images, filter implant, system noise like movement generated blur etc breeds to be carefully managed. This leads to the efficient reconstruction of High Resolution (HR) image made from the Low Resolution (LR) image. They also utilized the Kalman Filter (KF) to solve the computational problem to create an optimum Super Resolution context.

[2]. Chao Dong, Chen Change Loy, Kaiming He etc, explained in their paper 'Image Super-Resolution Using Deep Convolutional Networks' about a deep learning concept which was implanted with Super Resolution approach to increase the image resolution capabilities. It was designed for an end-to-end mapping of the low and high-resolution image. It used a deep convolution input image and straight away provide the output as a high-resolution image from its corresponding low-resolution image. Compared to the already available sparse Super Resolution concept this deep convolution concept used to take each layer and its corresponding elements separately. Unlike other methods, this was a lightweight concept to reduce the execution time. It was designed for real-time online implementations so this gave the system a cutting edge over other already available concepts. They went through different network structure to mitigate the execution speed and quality of output. They implemented the concept for 3 channel of colour and demonstrated the efficient quality of reconstruction.

[3] Saeideh Sarmadi and Zari Shamsa explained in their paper about single image super-resolution in which they deal with several problems faced during super-resolution. The major issues are solved systematic image processing of upscaling deblurring and demising. They have experimented the data for real-time results. At first, they have analyzed the image for lower resolution. This image needs to be reconstructed as a super-resolution image. Mainly super-resolution process needs the operation over blurring entity, dewnsampling entity and noise needed to be removed from the image. The denoising process deal with spatially adaptive iterative filtering (SAIF). This is a denoising technique which is most adaptive for local smoothening strength of the image based on the signal to noise (SNR) ratio analysis. Their technique was not very complex and clumsy ret efficiency enough to produce exceptional clarity. This made it a successful implementation. The system super-resolution developed by Saeideh Sarmadi and group provided clarity of understanding about the upscaling, deblurring and denoising process involved in super-resolution.

\section{SYSTEM ANALYSIS AND RESEARCH CONTENTS \& RESULTS}

The analysis is done over the image of lower resolution. We first reduced the quality of image shown in Fig. 1.2 by applying the blur as the addition parameter for the input image. This we do so we can analyse that for image with higher blur factors how the system behaves.
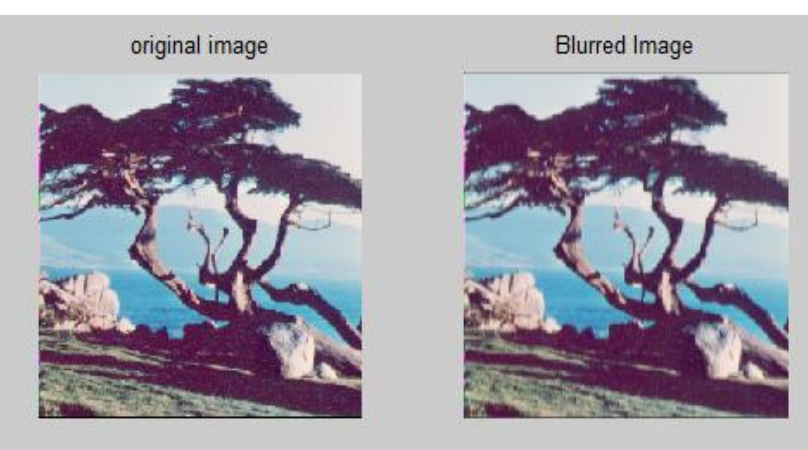

Fig. 1.2 - Super Resolution with Multiple Enhancements on base image

Then we selected the area which we want to Super Resolute. This gave the system a target Region of Interest (ROI) . This ROI can be selected by dragging the desired area.

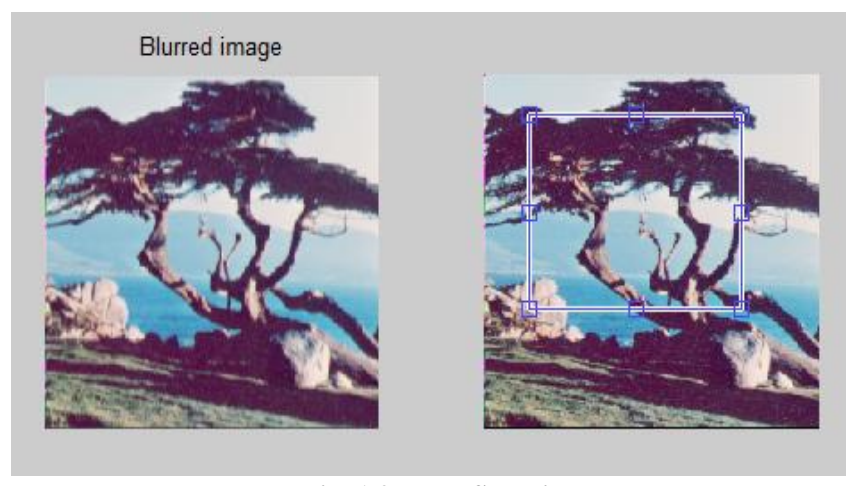

Fig. 1.3 - RoI Selection

The cropping feature extracts the part of image which we need to super resolute. This makes the system to efficiently allocate the area.
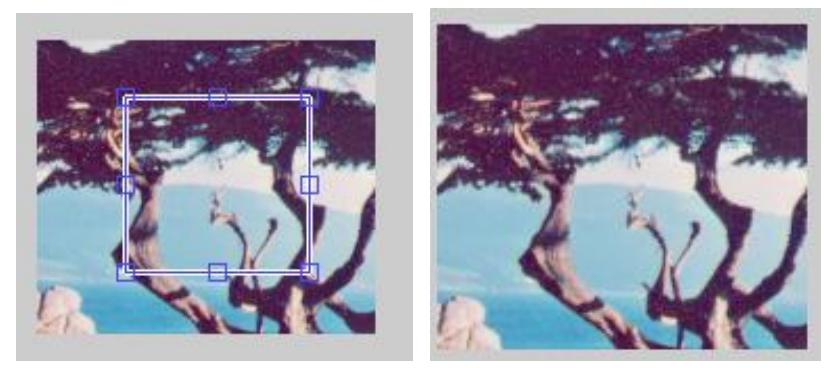

Fig. 1.4 - RoI Crop

The cropped region is the processed for deblurring , denoising and upscaling. This is the most important step of the process.

The final resulting step is the comparison of the results obtained. We can clearly analyse from the images displayed below that how the image is updcaled. We magnified the image to check the quality from ordinary system app and the result from our simlation. The result is displayed below. 


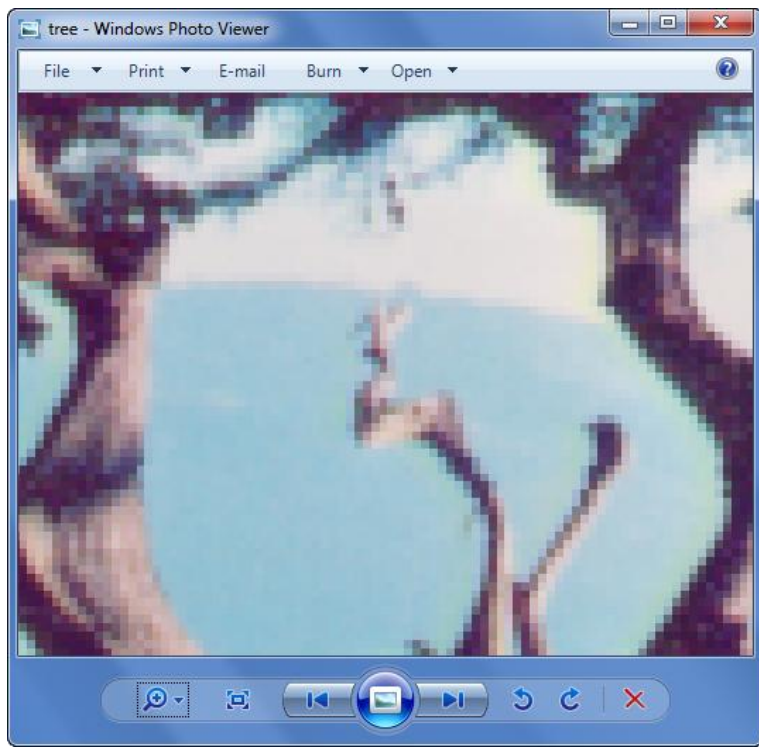

Fig. 1.5 - System Zoom

The above image of Fig. 1.5 is zoomed using the system picture viewer window app. This clearly shows that the image has the lower quality if it is zoomed exactly same as like the result image.

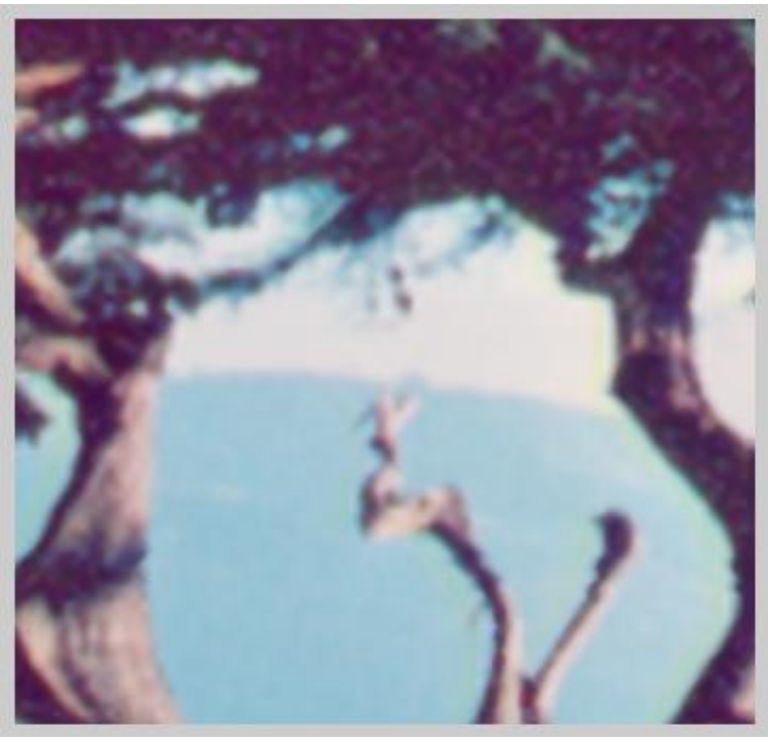

Fig. 1.6 - Super - Resolution Result

The above image of Fig. 1.6 is zoomed using Super Resolution upscaling system. This clearly shows that the image has the higher quality if it is zoomed exactly same as like the base image. The comparison clearly states that the Super Resolution image enhancement technique works perfectly even for the realtime images. This provides the most accurate result. It can be used reliably in different high quality imaging systems.

\section{CONCLUSION}

Obtaining a good resolution image is the prime requirement for almost every image processing and analysis based system. For better evaluation and synthesis of the contents involved in the image is a prime parameter. In most cases requirement of high-quality equipment to obtain exceptional results are mostly not fulfilled this makes the result less accurate and not reliable. In our paper, we came up with the ideology to have same quality hardware but with better conjunction of the image processing system. This provides a better resolution of the image after transforming the lower resolution image. The implementation concept deals with the process of super-resolution. The pixel enhancement is the key point for the overall system. As future conceptualization and implementation, it can be used to have zoom ability to be used in the broadest domain of space science. It can also be used in the microscopic domain like microbiology and nanotechnology.

\section{REFERENCE}

1. S. Wood, M Christensen, and D. Rajan, "Reconstruction algorithms for compound eye images using lens diversity,"paper presented at the Defense Applications of Signal Processing 2004 Workshop, Midway Utah, 27 March-1 April, 2005

2. S. L. Wood, B. J. Smithson, D. Rajan, and M. P. Christensen, "Performance of a MVE algorithm for compound eye image reconstruction using lens diversity," in Proceedings of IEEE International Conference on Acoustics, Speech, and Signal Processing, 2005 (ICASSP'05) (IEEE, 2005), pp. 593-596.

3. S. L. Wood, D. Rajan, M. P. Christensen, S. C. Douglas, and B. J. Smithson, "Resolution improvement for compound eye images through lens diversity," in Digital Signal Processing Workshop 2004 and the Third IEEE Signal Processing Education Workshop (IEEE, 2004), pp. 151-155, doi: 10.1109/DSPWS.2004.1437931.

4. H.-B. Lan, S. L. Wood, M. P. Christensen, and D. Rajan, "Benefits of optical system diversity for multiplexed image reconstruction," Appl. Opt. 45, $2859-2870$ (2006).

5. J. W. Goodman, Introduction to Fourier Optics, 2nd ed.(McGraw-Hill 1996), Chap. 6, pp. $146-151$.

6. H. L. Van Trees, Detection, Estimation, and Modulation Theory, Part I (Wiley, 1968).

7. A. W. Lohmann and W. T. Rhodes, "Two-pupil synthesis of optica transfer functions," Appl. Opt. 17, 1141-1151 (1978).

8. J. N. Mait and W. T. Rhodes, "Two-pupil synthesis of optical transfer functions. 2: Pupil function relationships," Appl. Opt., 2003-2007 (1986).

9. A. Macovski, Medical Imaging Systems (Prentice-Hall, 1983

10. C. Tomasi and R. Manduchi, "Bilateral filtering for gray and color images," in Proc. 6th Int. Conf. Compu. Vis., Ser., 1998, pp. 839-846.

11. C. Wang, L. Sun, P. Cui, J. Zhang, and S. Yang, "Analyzing image deblurring through three paradigms," IEEE Trans. Image Process., vol 21, no. 1, pp. 115-129, Jan. 2012.

12. N. Joshi, R. Szeliski, and D. Kriegman, "PSF estimation using sharp edge prediction," in Proc. IEEE Conf. Comput. Vis. Pattern Recognit., Jun. 2008, pp. 1-8.

13. A. Levin, Y. Weiss, F. Durand, and W. Freeman, "Efficient marginal likelihood optimization in blind deconvolution," in Proc. IEEE Comput. Soc. Conf. Comput. Vis. Pattern Recognit., Jun. 2011, pp. 2657-2664.

14. M. Almeida and L. Almeida, "Blind and semi-blind deblurring of natural images," IEEE Trans. Image Process., vol. 19, no. 1, pp. 36-52, Jan. 2010.

15. D. Keren, S. Peleg, and R. Brada, "Image sequence enhancement using sub-pixel displacements," in Proc. IEEE Comput. Soc. Conf. Comput. Vis. Pattern Recognit., Jun. 1988, pp. 742-746.

16. R. Lagendijk, J. Biemond, and D. Boekee, "Regularized iterative image restoration with ringing reduction," IEEE Trans. Acoustics, Speech Signal Process., vol. 36, no. 12, pp. 1874-1888, Dec. 1988.

17. M. Somayaji, M. P. Christensen, E. Faramarzi, D. Rajan, J.-P. Laine, P Sebelius, A. Zachai, M. Chaparala, G. Blasche, K. Baldwin, B. Ogunfemi, and D. Granquist-Fraser, "Prototype development and field-test results of an adaptive multiresolution PANOPTES imaging architecture,” Appl. Opt., vol. 51, no. 4, pp. A48-A58, Feb. 2012. 


\section{AUTHORS PROFILE}

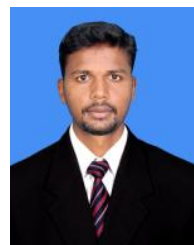

Mr.Durai Pandurangan (M.E) received his B.E (2009) in Electronics and Communication Engineering and M.E (2012) in Applied Electronics from Anna University, Tamilnadu, India. Durai Pandurangan is currently working as Lecturer in Department of Electrical and Computer Engineering, Wachemo University (WCU), Hossana, Ethiopia since Oct 2015

He has teaching experience of 8 years and advised 20 UG projects, co-advised 2 PG projects and published research papers on reputed journals and conferences. At WCU, he is working on the image enhancement techniques for weakly illuminated images. His area of interest includes Applied Electronics, Signal Processing, Communication Systems and Embedded Systems.

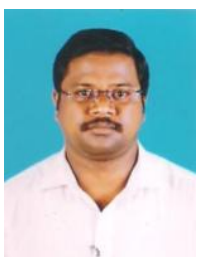

Mr.Ramakrishna MM, Pursuing $\mathrm{PhD}$ at Dr.M.G.R Educational and Research Institute in the field of Sensors has ten years of teaching Experience. He taught wide variety of courses in various engineering colleges of India and Ethiopia. He has graduated (B.E) in the field of Electronics and Instrumentation Engineering from University of Madras and also Post graduated (M.Tech) in the field of Instrumentation and Control Engineering from Bharath University. His research areas of interests are Sensors and Image Processing using MATLAB.

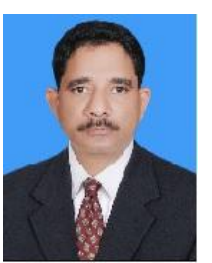

Mr. Gopala Krishna R (M Tech) received his B. E (1996) in Electronics and Communication Engineering and $\mathrm{M}$ Tech (1998) in Telecommunication Systems Engineering from IIT Kharagpur, India. Gopala Krishna is currently working as Lecturer in Department of Electrical and Computer Engineering, Wachemo University (WCU), Hossana, Ethiopia since Oct 2015 He has teaching experience of 15 years and industry experience of 2 years and advised number of academic projects and published research papers on reputed journals and conferences. At WCU, he is working on the image enhancement techniques for weakly illuminated images. His areas of interests include Digital Communications, Signal Processing and Embedded Systems.

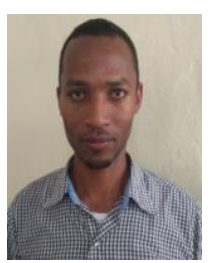

Mr.Alemayehu kebede received his B.E (2010) in Electrical Engineering from Mekele university and M.Tech in Microelectronics Engineering from Addis abebaUniversity,Ethiopia .Alemayehukebede is currently working as Lecturer in Department of Electrical and computer Engineering,Wachemo university (WCU), Hossana, Ethiopia since NOV 2014. He has teaching experience of 9 years and advised 35 UG projects. AtWCU, he is working on the image enhancement techniques for weakly illuminated images. His areas of interest includes applied electronics, embedded systems, wireless communication. 